\title{
A dark matter component decaying after recombination: lensing constraints with Planck data
}

\author{
Anton Chudaykin ${ }^{1,2, \star}$, Dmitry Gorbunov ${ }^{1,2, \star \star}$, and Igor Tkachev ${ }^{1,3, \star \star \star}$ \\ ${ }^{1}$ Institute for Nuclear Research of the Russian Academy of Sciences, Moscow 117312, Russia \\ ${ }^{2}$ Moscow Institute of Physics and Technology, Dolgoprudny 141700, Russia \\ ${ }^{3}$ Novosibirsk State University, Novosibirsk 630090, Russia
}

\begin{abstract}
It was recently proposed [1] that the model with a fraction of decaying cold dark matter is able to reconcile measurements in high redshift (CMB) and low redshift (probes of cluster abundance and the Hubble constant). We check this statement employing the full likelihood of CMB Planck data. We find that the lensing effect calculated from anisotropy spectra measured by Planck imposes the strong constraint on the fraction of unstable dark matter as $F<8 \%(2 \sigma)$. However, combining the CMB data with conflicting measurements in low redshift we obtain that the model with $F \approx 2-5 \%$ improves the goodness-of-fit by $1.5-2 \sigma$ depending on $A_{s}$ and $\tau$ priors in comparison with the concordance $\Lambda \mathrm{CDM}$ model.
\end{abstract}

\section{Introduction}

The nature of dark matter and dark energy in the Universe remains mysterious. Order-of-magnitude equality between contributions of baryonic matter and dark matter to the energy density of the Universe today is treated as a chance coincidence. In this situation dark matter may easily consists of several components. To distinguish them we suppose that one of that is unstable and decays into invisible radiation and the other behaviours as Cold Dark Matter (CDM).

In recent paper [1] the authors consider the model with a subdominant fraction of dark matter decaying after recombination and argued that such a model is able to reconcile the emerging tension between the cosmological probes in high redshift and low redshift. Namely, they reveal that the model under consideration explains extremely high value of the Hubble constant $h=0.738 \pm 0.024$ [2], $h=0.743 \pm 0.021$ [3] obtained in observations of astronomical Standard Candles in comparison with the corresponding value $h=0.6727 \pm 0.0066$ extracted from Planck spectra using TT, TE, EE + lowP dataset in notations of the original paper [4]. This model enable us to bring into accord constraints on parameters $\sigma_{8}, \Omega_{m}$ imposed by different Large Scale Structure tests with Planck observations as well. In our case, we check the consistency of the model with the galaxy cluster counts measured by Planck via Sunyaev-Zeldovich effect [4] which prefer lower values of $\sigma_{8}$.

However, in Ref. [1] the authors did not exploit the Planck likelihood properly. Instead of this they fixed all primary cosmological parameters relevant at recombination to Planck best-fit values

\footnotetext{
^e-mail: chudy@ms2.inr.ac.ru

$\star \star$ e-mail: gorby@ms2.inr.ac.ru

$\star \star \star$ e-mail: tkachev@ms2.inr.ac.ru
} 
- this approach guaranties that anisotropies at the last scattering will be identical in the Decaying Dark Matter (DDM) and $\Lambda \mathrm{CDM}$ models. Furthermore, the authors assume that the angular size of the sound horizon at the last scattering remains the same, or equivalently they put the quantity $\theta_{*}$ to the Planck value - this implies that CMB spectra calculated in DDM almost coincide with those obtained in $\Lambda \mathrm{CDM}$. A possible deviation from expected quantities can occur only due to gravitational distortions of spectra between last-scattering and the present epoch. Although such distortions are subdominant, the current cosmological data can be sensitive to them.

There are two sources of gravitationally distortions. The first one consists in the rise in the amplitude $C_{l}$ at low multipoles according to the integrated Sachs-Wolfe effect. This is caused by large values of the cosmological constant $\Lambda$ in spatially flat Universe since part of dark matter decays. This effect turns out to be insignificantly small giving the correction to $C_{l}$ below cosmic variance in the relevant range of multipoles (find explanation in [1]).

The second effect which was missed in [1] related to lensing of CMB anisotropies on the compact structure in the late Universe leaves imprints on observable CMB maps as well. The difference in lensing power in DDM and $\Lambda \mathrm{CDM}$ can be used as a smoking gun to resolve which model is better consistent with high quality data and provides better parameter convergence.

To fill the gap we fit all parameters of the DDM model to the current data accounting for the Planck likelihood properly. Our goal is to find out whether DDM is really able to reconcile cosmological measurements in high and low multipoles. We employ the complete Planck 2015 CMB data [5, 6], Hubble measurement [7] and the Planck cluster counts via Sunyaev-Zeldovich effect [8].

\section{The model, data sets and procedure}

\subsection{Decaying Dark Matter model}

The two component DDM model has two additional parameters, the fraction of decaying part in the total amount of dark matter, $F$, and the width of corresponding decay, $\Gamma$. We define the parameter $F$ in terms of initial quantities of stable and decaying dark matter density fractions as follows $F \equiv$ $\omega_{d d m} /\left(\omega_{s d m}+\omega_{d d m}\right)$. Initial means that these densities are measured if $\Gamma=0$. According to Ref. [1] we assume that unstable dark matter decays into massless invisible particles and the width $\Gamma$ is measured in units of $\mathrm{km} / \mathrm{s} / \mathrm{Mpc}$ as $H_{0}$.

\subsection{Cosmological data sets}

The main cosmological dataset we always used here is TT,TE,EE which include all CMB spectra measured by Planck in the multipole range $l>30$ in full compliance with [5]. It already contains gravitational distortions of CMB anisotropies due to lensing on compact structures in the late Universe. Lensing results in smoothing acoustic oscillation in the CMB spectra, so the peaks become lower and the troughs higher.

In some cases we include information about $\mathrm{CMB}$ anisotropies at low multipoles including polarisation data and refer to this set as lowP in later according to [5].

Since the lensing effect is the most critical for us we also include the direct measurement of the lensing potential $C_{l}^{\phi \phi}$ computed by Planck Collaboration using non-Gaussian (connect) part of 4-point correlation functions [6]. We use the notation lens for this measurement.

For the low redshift measurements we employ the constraints on the present expansion rate of the Universe and on abundance of Large Scale Structure today. Namely, we include the current measurement of the Hubble constant with smaller error recently updated by Riess et. al [7] and denote it as $\mathrm{H}_{0}$. 


\begin{tabular}{|c|}
\hline Dataset \\
\hline$\left(\mathrm{TT}, \mathrm{TE}, \mathrm{EE}+\mathrm{H}_{0}+\mathrm{CL}\right)+$ lowP \\
$\left(\mathrm{TT}, \mathrm{TE}, \mathrm{EE}+\mathrm{H}_{0}+\mathrm{CL}\right)+$ lens \\
$\left(\mathrm{TT}, \mathrm{TE}, \mathrm{EE}+\mathrm{H}_{0}+\mathrm{CL}\right)+$ lowP + lens \\
\hline
\end{tabular}

Table 1. Datasets used in our analysis.

As for structure tests we adopt the galaxy cluster counts measured by Planck via Sunyaev-Zeldovich effect which was used in Ref. [1] as well. We indicate this Planck probe below as CL.

Throughout the paper we always use the data combination (TT, TE, EE $+\mathrm{H}_{0}+\mathrm{CL}$ ). However, the measurement of lensing amplitude obtained here is in moderate conflict with one extracted from lens, see $[5,6]$. Therefore we decide to separate these probes of the lensing effect at late times and divide all data into three blocks: with lowP, lens and lowP+lens. Our data separation reflects the main difference in lensing power predicted by various cosmological observations.

\subsection{Numerical procedure}

To find the allowed region in DDM model parameter space we carry out the Markov Chain Monte Carlo analysis using the Monte Python package [9] with the CLASS Boltzman code [10,11] adopted to the two component DDM model. Eight parameters have been fitted to various observational data: two of them describe intrinsic properties of the DDM model, the fraction $F$ and the width $\Gamma$, whereas the remaining six - the angular size of the sound horizon $r_{s}$ at last-scattering $\theta_{*} \equiv 100 \times r_{s}\left(z_{*}\right) / D_{A}\left(z_{*}\right)$, the baryon density $\omega_{b}$, initial CDM density $\omega_{c d m}=\omega_{s d m}+\omega_{d d m}$, the optical depth $\tau$, the squared amplitude $A_{s}$ and tilt $n_{s}$ of the power spectrum of primordial scalar perturbations - are standard. In our numerical analysis we calculate the perturbations in linear regime only preliminary checked that nonlinear corrections to all relevant quantities are rather small in our approach. For instance, the nonlinear contribution to the matter power spectrum is below $1 \%$ at scales relevant for estimation the parameter $\sigma_{8}$. We consider the Universe is spatially flat, neglect possible tensor perturbations in metric and assume normal hierarchy pattern with the sum of the active neutrino masses $m_{v}=0.06 \mathrm{eV}$.

\section{Constraints on DDM}

\subsection{Planck data only}

Firstly, we emphasize the particular role of the lensing effect which affects the TT power spectrum. For this purpose we depict the difference in this quantity between DDM $(F=0.1, \Gamma=2000 \mathrm{~km} / \mathrm{s} / \mathrm{Mpc})$ and $\Lambda \mathrm{CDM}$ predictions assuming the other parameters are fixed to the best-fit values obtained in TT, TE, EE + lowP analysis. The corresponding difference is shown in the left-hand panel of Fig. 1 by solid red line. The Planck data with the preliminary subtracted prediction of $\Lambda \mathrm{CDM}$ is depicted on the same plot as blue points with error bars as well. We see that the Planck's sensitivity and the difference in lensing power between DDM and $\Lambda$ CDM models are comparable to each other; this enables the data to feel the peculiar feature of DDM - different lensing power. Although the lensing effect is accounted for in both models, the prediction of the concordance $\Lambda$ CDM model does not correspond the data points perfectly: the data points oscillate closely to zero. This means that the Planck likelihood prefers a bit stronger lensing effect in comparison with the theoretical prediction of 
$\Lambda \mathrm{CDM}$ model; corresponding disagreement is at $2 \sigma$ confidence level [6]. Although the difference in best-fit predictions of DDM and $\Lambda \mathrm{CDM}$ models (red solid line) is comparable with deviation of the residuals of the data (blue points), it is out of phase. This reflects lensing power even weaker than in the standard cosmology model. As a result of that, proper fitting to TT, TE, EE + lowP likelihood impose the strong constraint on the parameter of DDM, $F<0.07(2 \sigma)$. This observation was missed in the previous work [1].

To emphasize the role of the lensing effect and to reveal the importance of that more explicitly in our approach we show the difference in the lensing power spectrum between $\operatorname{DDM}(F=0.1, \Gamma=$ $2000 \mathrm{~km} / \mathrm{s} / \mathrm{Mpc}$ ) and $\Lambda \mathrm{CDM}$ predictions for the same best-fit parameters obtained in the TT, TE, EE + lowP analysis. The solid red curve in the right panel of Fig. 1 depicts this difference whereas the data
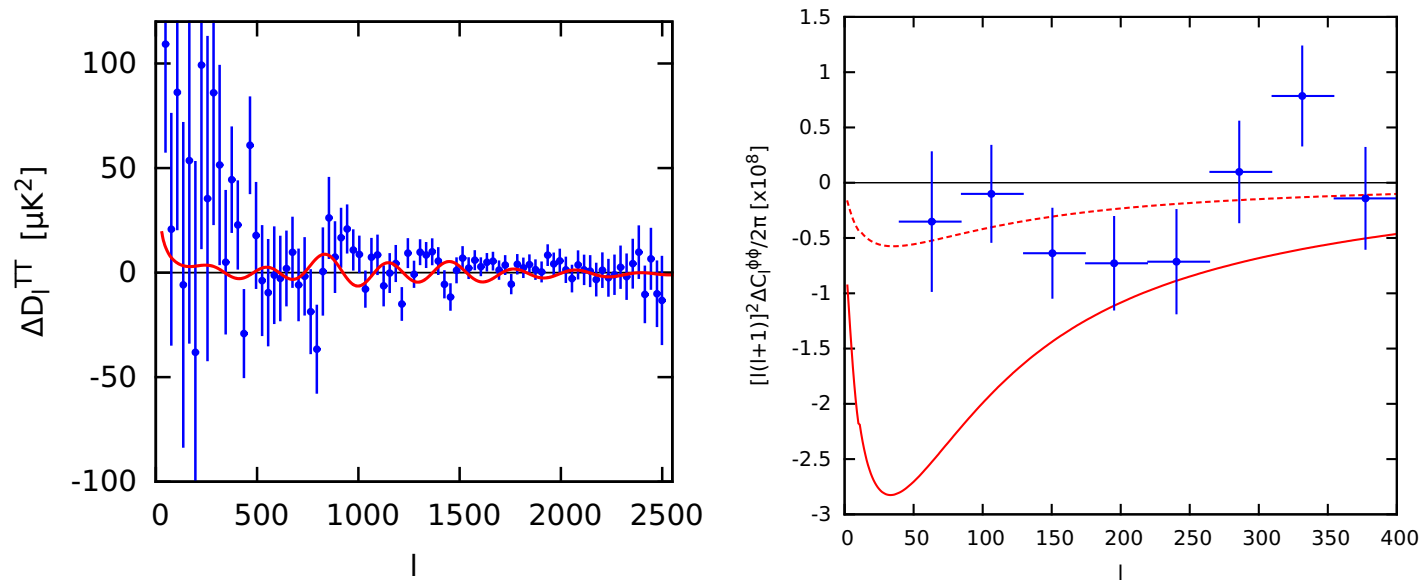

Figure 1. Left. Data points with error bars show residuals after subtraction from the measured TT power spectrum the $\Lambda \mathrm{CDM}$ prediction with the best-fit parameters obtained in TT, TE, EE + lowP analysis. Solid curve corresponds to the difference between TT spectra in DDM $(F=0.1, \Gamma=2000 \mathrm{~km} / \mathrm{s} / \mathrm{Mpc})$ and $\Lambda$ CDM for the same best-fit parameters. Right. Data points with error bars show residuals after subtraction from the directly measured lensing power spectrum by Planck the best-fit $\Lambda$ CDM prediction within TT, TE, EE + lowP analysis. The solid curve depicts the difference in $C_{l}^{\phi \phi}$ between DDM $(F=0.1, \Gamma=2000 \mathrm{~km} / \mathrm{s} / \mathrm{Mpc})$ and $\Lambda C D M$ models assuming the same values of standard parameters as in the left panel. The dotted curve corresponds to the best-fit DDM model obtained in the TT, TE, EE + lowP + lens analysis.

points with error bars correspond to the direct measurement of the lensing power spectrum computed from the connected 4-point functions by Planck Collaboration after subtraction the best-fit $\Lambda$ CDM model from the data. As we can see from the right-hand plot of Fig. 1 lensing power in DDM is actually weaker than in the standard cosmology as mentioned above. However the DDM model pushes the $C_{l}^{\phi \phi}$ values towards the prediction of the lens dataset. Therefore the model with DDM is worse consistent with Planck data spectrum and better consistent with the direct measurement of the lensing power spectrum than the model with CDM. Because of the first statement the DDM model is tightly constrained by TT, TE, EE likelihood and because of the second one - taking into account lens data provides a more conservative constraint on the parameter $F$.

The data set lowP does not restrict lensing power directly. However, the situation is a little more tricky and the role of the polarisation measurement at low multipoles coupled to the TT, TE, EE probe turns out to be important. The lack of lensing power in DDM causes higher amplitude values of primordial scalar spectra, $A_{s}$, which, in turn, amplifies the amplitude of $C_{l}^{T T}$. To compensate the latter 
growth larger values of optical depth, $\tau$, is needed but the quantity $\tau$ is strongly constrained by lowP likelihood. As a result, the parameter $F$ becomes even more restricted in combined TT, TE, EE + lowP dataset, $F<0.04(2 \sigma)$, in comparison with the prediction of TT, TE, EE data only.

The preference of the lens data is opposite. The direct measurement of $C_{l}^{\phi \phi}$ prefers a little bit weaker lensing power as compared to the $\Lambda \mathrm{CDM}$ prediction $[5,6]$. It means that lens likelihood favours DDM consideration rather then $\Lambda \mathrm{CDM}$ one and causes the weaker constraint on the DDM parameter, $F<0.08(2 \sigma)$.

\subsection{Planck data and conflicting low z measurements}

Now we combine the Planck probes in high redshift with the measurements of $H_{0}, \Omega_{m}$ and $\sigma_{8}$ in low redshift. Since the DDM is restricted by lensing which acts in opposite directions in different cosmological observations we explore the parameter space in DDM using three different cosmological datasets which are listed in Table 1.

The corresponding constraints on DDM parameters and on the relevant quantities in low redshift measurements are plotted in Figs. 2 - 3. One can see from Fig. 2 that the preferred values $F \simeq 2-5 \%$

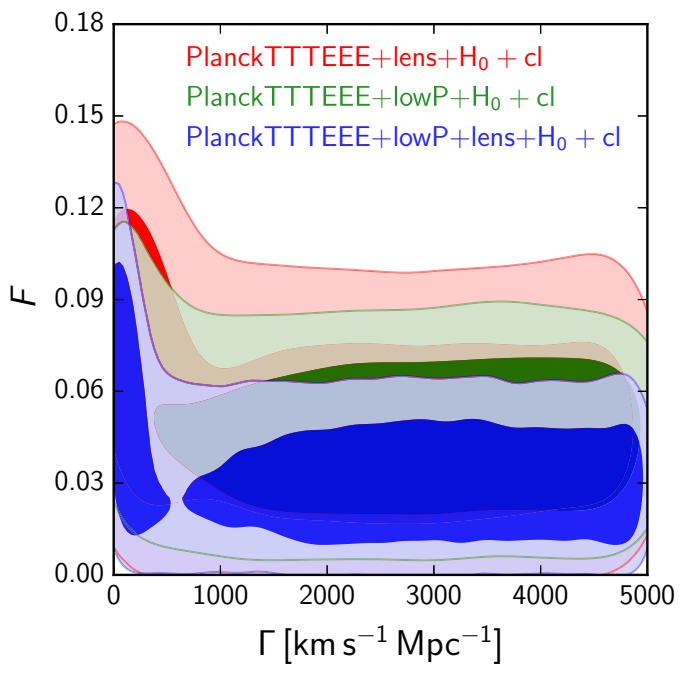

Figure 2. Posterior distributions ( $1 \sigma$ and $2 \sigma$ confident regions) of parameters $F$, $\Gamma$ in the DDM model.

are almost independent of the parameter $\Gamma$ in the range $1000 \mathrm{~km} /(\mathrm{s} \mathrm{Mpc}) \lesssim \Gamma \lesssim 5000 \mathrm{~km} /(\mathrm{s} \mathrm{Mpc})$ which means that all amount of unstable dark matter completely decays after recombination since $\Gamma \gg H_{0}$. We emphasize that the line $F=0$ which corresponds to the models with a minuscule fraction of DDM is within $2 \sigma$ confident region for the (TT, TE, EE $+\mathrm{H}_{0}+\mathrm{CL}$ ) + lens and (TT, TE, EE $+\mathrm{H}_{0}+$ $\mathrm{CL})+$ lowP + lens datasets, but outside for (TT, TE, EE $\left.+\mathrm{H}_{0}+\mathrm{CL}\right)+$ lowP. The increase in $F$ for lower values of $\Gamma$ corresponds to the models with non vanishingly small amount of DDM today. In the limit $\Gamma \ll H_{0}$, DDM at the present is undistinguishable from common CDM. The regions of small $\Gamma$ is unresolved here and deserve a special study beyond the current research. In the left-hand panel of Fig. 3 the allowed regions at $2 \sigma$ confidence level with highest values of $F$ and lowest values of $H_{0}$ correspond to the range $\Gamma \ll 1000 \mathrm{~km} /(\mathrm{s} \mathrm{Mpc})$ in Fig. 2 with long-lived DDM particles. In the 


\begin{tabular}{|c|c|c|c|}
\hline dataset & $\Delta \chi^{2}$ & p-value & improvement \\
\hline (TT, TE, EE $\left.+\mathrm{H}_{0}+\mathrm{CL}\right)+$ lowP & 5.34 & 0.0692 & $1.81 \sigma$ \\
(TT, TE, EE $\left.+\mathrm{H}_{0}+\mathrm{CL}\right)+$ lens & 4.68 & 0.0963 & $1.66 \sigma$ \\
$\left(\mathrm{TT}, \mathrm{TE}, \mathrm{EE}+\mathrm{H}_{0}+\mathrm{CL}\right)+$ lowP + lens & 4.28 & 0.1176 & $1.56 \sigma$ \\
\hline
\end{tabular}

Table 2. Improvement of DDM over $\Lambda$ CDM in three datasets considered and listed Tab. 1 in case of 2 extra degrees of freedom in DDM.

right panel of Fig. 3 the regions with highest allowed values of $\sigma_{8}$ and lowest values of $\Omega_{m}$ map to the parameter space $1000 \mathrm{~km} /(\mathrm{s} \mathrm{Mpc}) \lesssim \Gamma \lesssim 5000 \mathrm{~km} /(\mathrm{s} \mathrm{Mpc})$ and highest values of $F$ in Fig. 2 .
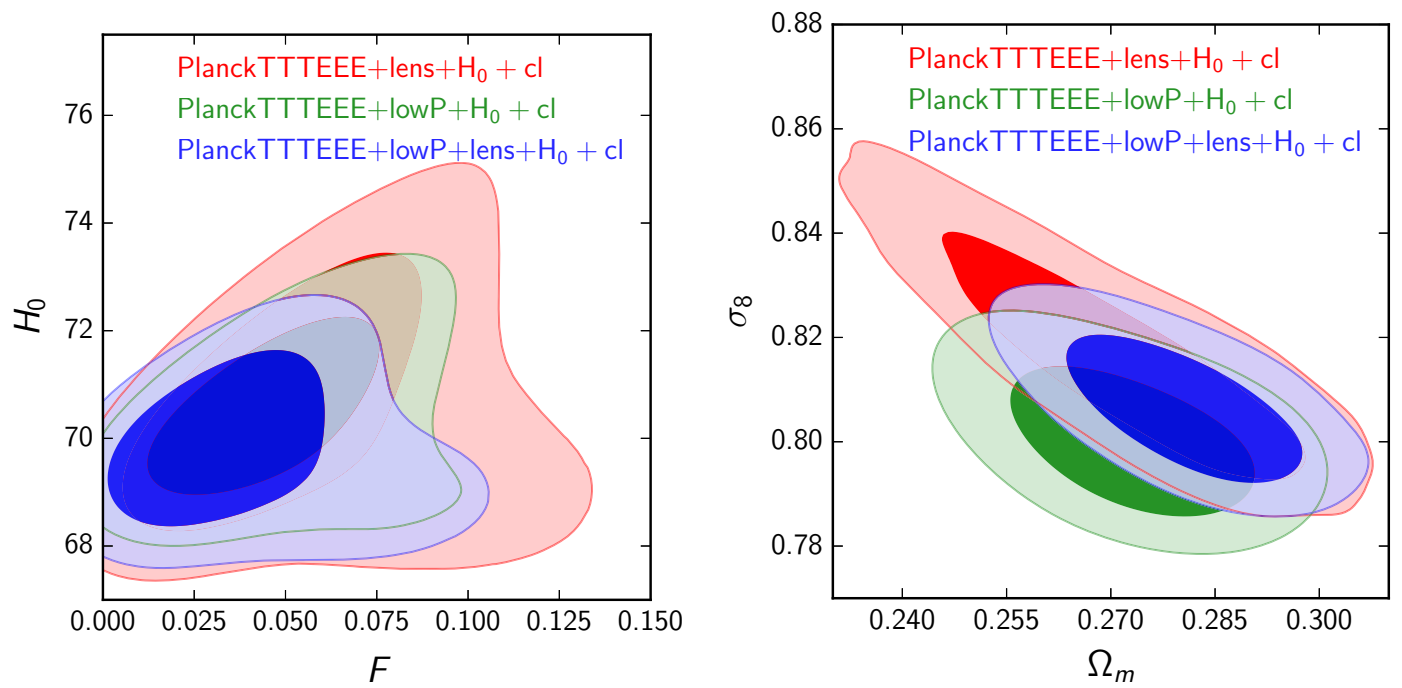

Figure 3. Same as Fig. 2 but for pairs of the parameters $H_{0}-F$ and $\sigma_{8}-\Omega_{m}$.

We notice that proper fitting gives significantly stronger upper limit on the fraction $F$ which contradicts the result obtained in Ref. [1]. In addition, the allowed region for parameters $F, \Gamma$ obtained in Ref. [1] is well outside the $2 \sigma$ contours depicted in Fig. 2. However, the DDM consideration still prefers non-zero values of the parameter $F$ and absence of the decaying part of dark matter here is disfavoured.

To understand which model better describes various observational data we compare logarithmic likelihood $\log L$ calculated for both models in their best-fit points. The difference $2 \cdot \Delta \log L$ between DDM and $\Lambda \mathrm{CDM}$ is distributed as $\chi^{2}$ with effective number of degree of freedom equalled to the difference in fitting parameters in these two models. In our research the number of fitting parameters equals 2 and corresponds to the DDM parameters, $F$ and $\Gamma$. The results of such quantitative analysis are represented in Tab 1.

The DDM consideration indeed improves the goodness-of-fit in comparison with $\Lambda \mathrm{CDM}$ one as suggested in Ref. [1]. However the improvement is not so significant as suggest before because the 
Planck data suffers from the lensing conflict represented in Fig. 1. It is entered in all datasets and is worse for DDM than for $\Lambda \mathrm{CDM}$.

In principle, the corresponding constraint of $F$ is able to rule out DDM completely in future. But we caution the reader while we do not understand the origin of the lensing conflict within one precise measurement we are not able to make any strong conclusion about favour or disfavour of the two component DDM model in comparison with $\Lambda$ CDM one.

\section{Conclusion}

We confirm that the DDM consideration provides better description of the current observational data which consists of the precise Planck probe of the Universe at hight redshifts and measurements of the cosmological parameters at low redshifts. However, the favoured values of the fraction $F$ are significantly smaller than ones claimed in the previous work [1].

We explain why lensing information is critical for verification of the two component DDM model using the current cosmological data. Planck measures lensing power in two different ways. Firstly, lensing is extracted from CMB anisotropy maps as smoothing the acoustic structure of the spectra. The second probe of lensing power consists in the direct measurement of the lensing power spectrum $C_{l}^{\phi \phi}$ using nun-Gaussian parts of all 4-point correlation functions. But both these measurements are conflicting with the $\Lambda \mathrm{CDM}$ prediction which could be also treated as disagreement between low- $\mathrm{Z}$ and high-z experiments. Namely, the concordance $\Lambda \mathrm{CDM}$ model predicts a bit stronger lensing power as compared to the direct measurements of $C_{l}^{\phi \phi}$ and significantly weaker one in comparison with the preference of TT, TE, EE + lowP likelihood. Therefore the former data actually favours DDM whereas the latter tightly constrains.

Taking into account the lensing conflict inside the Planck likelihood, we have checked whether the DDM model is able to reconcile measurements at high and low redshifts. The improvement of DDM over $\Lambda \mathrm{CDM}$ is observed in our research but this is not so prominent as suggested before. We feel that the lensing information extracted from the current data is contradictory and requires further investigation. This means that arguments based on present lensing information only should not be taken until the lensing conflict in the Planck data has not been totally resolved.

We extend gratitude to Z. Berezhiani, A. Dolgov and J. Lesgourgues for helpful discussion of the material. The work of A. Chudaykin and D. Gorbunov has been supported by Russian Foundation for Basic Research grant 14-02-00894. All simulations in the paper are carried out with MVS-10P supercomputer of Joint Supercomputer Center of the Russian Academy of Sciences (JSCC RAS).

\section{References}

[1] Z. Berezhiani, A.D. Dolgov, I.I. Tkachev, Phys. Rev. D92, 061303 (2015), 1505.03644

[2] A.G. Riess, L. Macri, S. Casertano, H. Lampeitl, H.C. Ferguson, A.V. Filippenko, S.W. Jha, W. Li, R. Chornock, Astrophys. J. 730, 119 (2011), [Erratum: Astrophys. J.732,129(2011)], 1103.2976

[3] W.L. Freedman, B.F. Madore, V. Scowcroft, C. Burns, A. Monson, S.E. Persson, M. Seibert, J. Rigby, Astrophys. J. 758, 24 (2012), 1208.3281

[4] P.A.R. Ade et al. (Planck) (2015), 1502.01597

[5] P.A.R. Ade et al. (Planck) (2015), 1502.01589

[6] P.A.R. Ade et al. (Planck) (2015), 1502.01591

[7] A.G. Riess et al., Astrophys. J. 826, 56 (2016), 1604.01424

[8] P.A.R. Ade et al. (Planck), Astron. Astrophys. 571, A20 (2014), 1303.5080 
[9] B. Audren, J. Lesgourgues, K. Benabed, S. Prunet, JCAP 1302, 001 (2013), 1210.7183 [10] J. Lesgourgues (2011), 1104 . 2932

[11] D. Blas, J. Lesgourgues, T. Tram, JCAP 1107, 034 (2011), 1104 . 2933 